\title{
Respiratory depression as side effect: should benzodiazepines be avoided in patients with COVID-19?
}

\author{
Sander Borgsteede ${ }^{1}$, Klaas Van Kralingen ${ }^{2}$, and Janwillem Kocks ${ }^{3}$ \\ ${ }^{1}$ Health Base Foundation \\ ${ }^{2}$ Sleep Wake Centre SEIN \\ ${ }^{3}$ General Practitioners Research Institute
}

May 6, 2020

\begin{abstract}
The pharmacological profile of benzodiazepines includes respiratory depression, and theoretically benzodiazepines might contribute to respiratory dysfunction. There are currently no data that indicate that benzodiazepines are problematic in COVID-19, and the risk is expected to be limited. Benzodiazepines are effective and frequently used, and if there is a clear indication in patients with COVID-19 there is insufficient reason to avoid benzodiazepines based on respiratory depression as side effect.
\end{abstract}

Several medications such as ACE-inhibitors have been associated with a negative influence on COVID-19 based on their potential pharmacological activity to interfere with viral cell invasion, however the causality and clinical relevance have not been proven yet and this is subject of investigation.[1] At our helpdesk for clinical decision support, we received questions from health care professionals if benzodiazepines could negatively influence the course of non-intubated COVID-19 patients by their side effects, and hence should better be avoided. Benzodiazepines are frequently used for indications such as sleep and anxiety, also in seriously ill patients. In this letter, we comment on the relevance of respiratory depression as side effect of benzodiazepines in patients with COVID-19 from a pharmacological and epidemiological perspective.

Benzodiazepines are $\mathrm{GABA}_{\mathrm{A}}$-agonists, and respiratory depression is a well-known side effect mentioned in the official product information. Their main effect on respiratory function is depression of the central respiratory drive and lowering of the responsiveness to hypercapnia.[2] Other hypothesised pathways include direct activation of $\mathrm{GABA}_{\mathrm{A}}$-receptors in the peripheral nervous system resulting in reduced respiratory muscle strength, and contribution to local inflammation.[2] However, the findings on respiratory depression in humans are inconclusive and contradictory, and it is unclear how benzodiazepines are involved in the pathways behind these processes.[3]

From an epidemiological perspective, the use of high (but not low) dose benzodiazepines in patients with severe (oxygen dependent) COPD increases the risk for mortality, but prospective trials have not shown effects on gas exchange or breathing rate.[4] Epidemiological studies are hampered by residual confounding, and therefore they might overestimate the effect of benzodiazepines, yet these studies advise in agreement with prescribing guidelines to be cautious with benzodiazepines, especially when co-prescribed with opioids.[5] In end-of-life care, the use of benzodiazepines can be indicated for anxiety or the relief of dyspnoea.[6,7]

In the course of COVID-19, benzodiazepines are feared to worsen the respiratory function in patients who experience shortness of breath in the second week of illness, that may further develop to acute respiratory distress syndrome in about 10-20\% of the severe patients. [8] However, as the respiratory problems in COVID19 are caused by a type 1 respiratory failure, and as the risk of benzodiazepines is predominantly associated 
with hypercapnia, the expected risk is limited. However, future epidemiological analyses that will investigate the use of medication as factor contributing to the course of COVID-19, should also study the use of benzodiazepines.

In conclusion, the pharmacological profile of benzodiazepines includes respiratory depression, and theoretically benzodiazepines might contribute to respiratory dysfunction. There are currently no data that indicate that benzodiazepines are problematic in COVID-19, and the risk is expected to be limited. Benzodiazepines are effective and frequently used, and if there is a clear indication in patients with COVID-19 there is insufficient reason to avoid benzodiazepines based on respiratory depression as side effect.

\section{References}

1. Vaduganathan M, Vardeny O, Michel T, McMurray JJV, Pfeffer MA, Solomon SD. Renin-AngiotensinAldosterone System Inhibitors in Patients with Covid-19. N Engl J Med. 2020 Mar 30. doi: 10.1056/NEJMsr2005760.

2. Vozoris NT. Do benzodiazepines contribute to respiratory problems? Expert Rev Respir Med. 2014 Dec;8(6):661-3. doi: 10.1586/17476348.2014.957186.

3. Masneuf S, Buetler J, Koester C, Crestani F. Role of $\alpha 1$ - and $\alpha 2$-GABA(A) receptors in mediating the respiratory changes associated with benzodiazepine sedation. Br J Pharmacol. 2012 May;166(1):33948. doi:10.1111/j.1476-5381.2011.01763.x.

4. Ekström MP, Bornefalk-Hermansson A, Abernethy AP, Currow DC. Safety of benzodiazepines and opioids in very severe respiratory disease: national prospective study. BMJ 2014; 348: g445.

5. UK Medicines \& Healthcare products Regulatory Agency. Benzodiazepines and opioids: reminder of risk of potentially fatal respiratory depression. Drug Safety Update volume 13, issue 8: March 2020: 5.

6. Salt S, Mulvaney CA, Preston NJ. Drug therapy for symptoms associated with anxiety in adult palliative care patients. Cochrane Database Syst Rev. 2017 May 18;5:CD004596. doi: 10.1002/14651858.CD004596.pub3.

7. Kloke M, Cherny N; ESMO Guidelines Committee. Treatment of dyspnoea in advanced cancer patients: ESMO Clinical Practice Guidelines. Ann Oncol. 2015 Sep;26 Suppl 5:v169-73. doi: 10.1093/annonc/mdv306.

8. Zhou M, Zhang X, Qu J. Coronavirus disease 2019 (COVID-19): a clinical update. Front Med. 2020 Apr 2. doi: 10.1007/s11684-020-0767-8. [Epub ahead of print] 